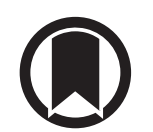

CrossMark

\title{
Association between age and clinical characteristics and outcomes of COVID-19
}

\author{
To the Editor:
}

The rapid outbreak of coronavirus disease 2019 (COVID-19) has been a matter of international concern as the disease is spreading fast $[1,2]$. Considering that the contagious disease has led to an enormous impact globally, there is an urgent need to identify the risk populations with poor prognosis. Ageing is associated with certain changes in pulmonary physiology, pathology and function, during the period of lung infection. Therefore, age-related differences in responsiveness and tolerance become obvious and lead to worse clinical outcomes in elderly individuals [3]. Previous studies have mentioned that older COVID-19 patients are at an increased risk of death [4-7]. However, the age-related clinical characteristics, disease courses and outcomes other than death in COVID-19 patients remain unclear.

In total, 221 COVID-19 patients who were diagnosed by the fever clinics of the designated hospitals were included in this study. They were administratively admitted to Shanghai Public Health Clinical Center (Shanghai, China) to receive medical care. The diagnosis was based on a positive response to viral nucleic acid detection, according to the updated version of the guidelines for the diagnosis and treatment of COVID-19 pneumonia issued by the National Health Commission of China [8]. Data on demographics, symptoms, disease severity and course, radiological and laboratory examination were analysed in our study. A unified observation end-point date was set in our study (7 March 2020). For all COVID-19 patients, the primary outcome of disease course and the secondary outcome of respiratory failure rate were compared in two age groups.

All 221 COVID-19 patients were divided into two groups, taking age 60 years as the threshold. The age distribution for all patients is shown in figure 1a. 136 cases $(61.5 \%)$ were aged $<60$ years and the other cases were aged $\geqslant 60$ years. In total, 176 patients $(79.6 \%)$ had fever. Other common symptoms included cough $(48.0 \%)$, sputum $(25.8 \%)$, sore throat $(8.6 \%)$ and diarrhoea $(5.4 \%)$, among which only sore throat showed a significant difference between the two groups $(11.8 \%$ versus $3.5 \% ; \mathrm{p}=0.034)$. Significant negative correlations between age of patients and lymphocyte counts $(\mathrm{r}=-0.432, \mathrm{p}<0.001)$ as well as albumin levels $(\mathrm{r}=-0.569, \mathrm{p}<0.001)$ were observed in our study (figure $1 \mathrm{~b}$ and $\mathrm{c}$ ). Compared with the younger group, patients aged $\geqslant 60$ years presented with higher levels of blood urea nitrogen (5.83 versus $4.42 \mathrm{mmol} \cdot \mathrm{L}^{-1}$; $\mathrm{p}<0.001)$, lactate dehydrogenase $\left(272.8\right.$ versus $\left.249.0 \mathrm{U} \cdot \mathrm{L}^{-1} ; \mathrm{p}=0.004\right)$ and inflammatory indicators (figure $1 \mathrm{~d}$; all $\mathrm{p}<0.01$ ). In those aged $\geqslant 60$ years, more lobes were involved $(4.15$ versus $3.34 ; \mathrm{p}<0.001)$ in bilateral lesions $(89.4 \%$ versus $74.3 \%$; $\mathrm{p}=0.006$ ) (figure $1 \mathrm{e}$ ) and a higher proportion of patients showed bacterial co-infection ( $12.9 \%$ versus $4.4 \%$; $=0.021)$. The severity of COVID-19 was milder in those aged $<60$ years; this younger group showed lower proportions of severe and critical patients (figure 1f). In turn, the utilisation of antibiotic therapy, intravenous corticosteroids and assisted ventilation was more common in those aged $\geqslant 60$ years (figure $1 \mathrm{~g}$ ). Longer disease courses and a higher proportion of cases with respiratory failure were observed in patients aged $\geqslant 60$ years (figure $1 \mathrm{~h}$ ). The median time of disease course was significantly longer in patients aged $\geqslant 60$ years $(24.0$ versus 21.5 days; $\mathrm{p}=0.026)$ (figure $1 \mathrm{i})$. Interestingly, this difference was markedly significant in male patients ( 25.0 versus 21.0 days; $\mathrm{p}=0.036)$ but not in female patients (figure $1 \mathrm{j}$ and $\mathrm{k}$ ). A big difference in the median time of disease course was observed between the two age groups in cases with respiratory failure, although it showed no statistical significance (38.0 versus 30.0 days; $\mathrm{p}=0.100$ ) (figure 11 ).

@ERSpublications

Age significantly determined the clinical features and prognosis of COVID-19. The prognosis was worse in patients older than 60 years, calling for clinicians to pay more attention to patients of this age. https://bit.ly/34DTI05

Cite this article as: Liu Y, Mao B, Liang S, et al. Association between age and clinical characteristics and outcomes of COVID-19. Eur Respir J 2020; 55: 2001112 [https://doi.org/10.1183/13993003.01112-2020]. 

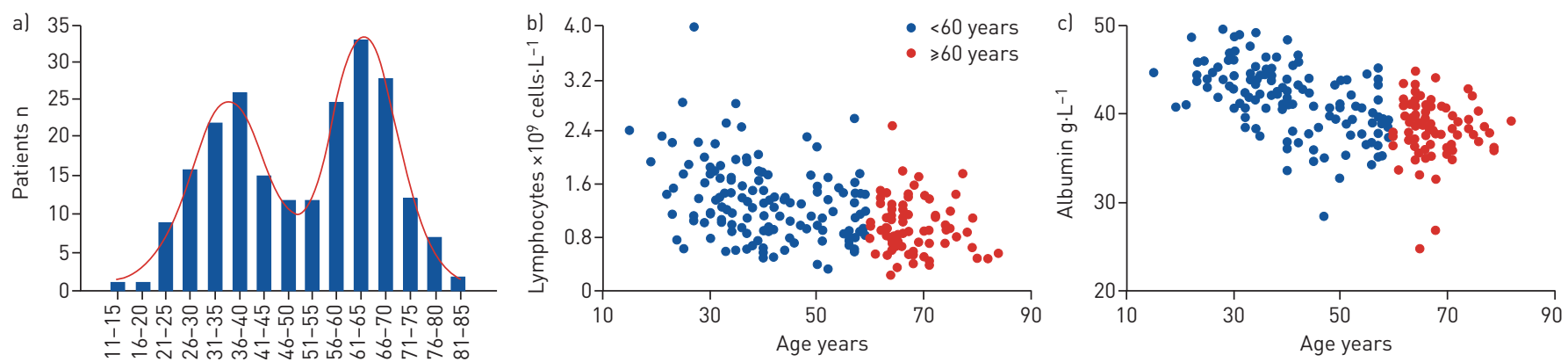

Age years
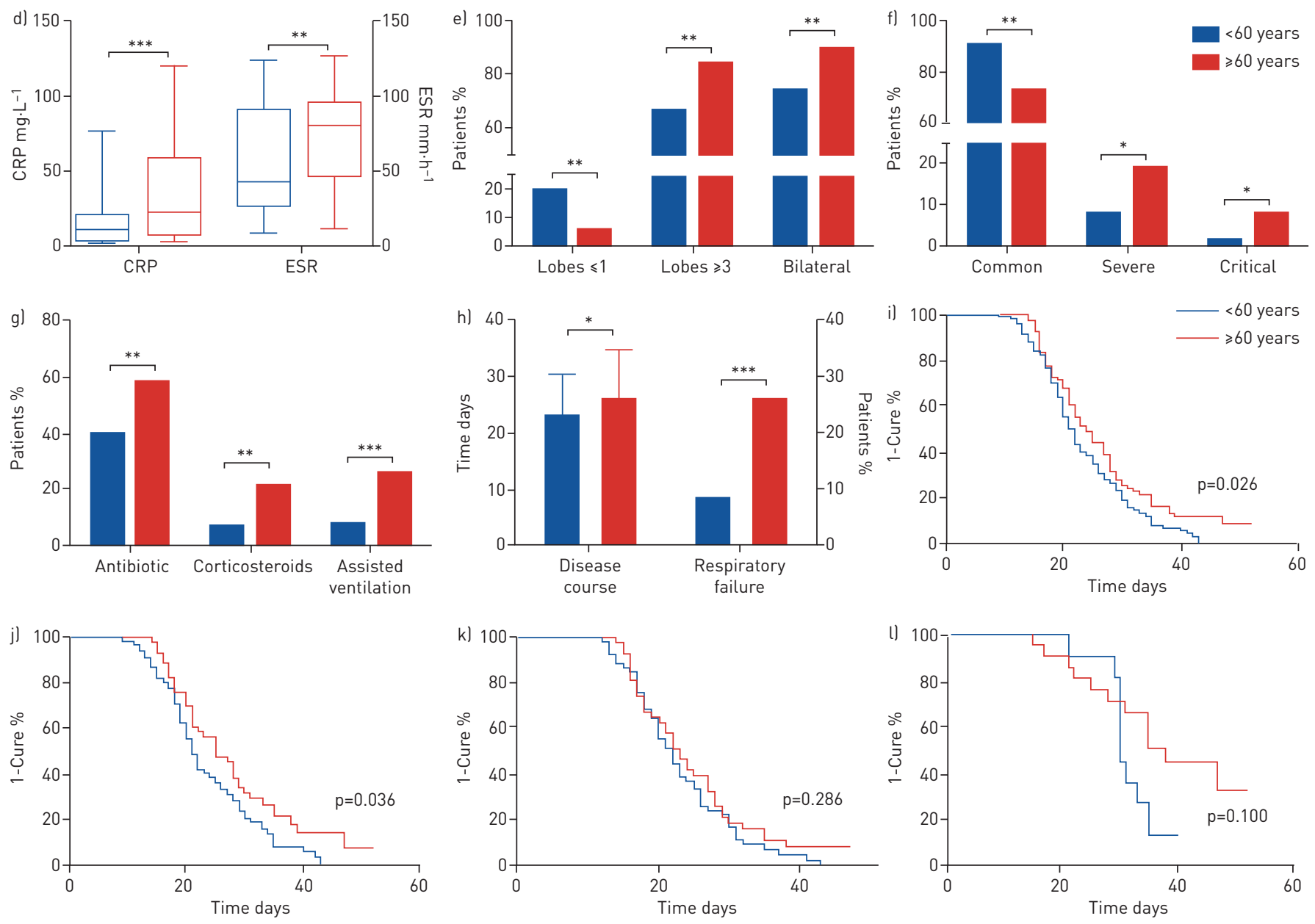

FIGURE 1 Comparison of clinical features and outcomes of COVID-19 between two age groups, $<60$ and $\geqslant 60$ years. a) Age distribution for all 221 COVID-19 patients in Shanghai. Correlations were investigated between the age of patients and $b)$ lymphocyte count ( $r=-0.432, p<0.001)$, c) albumin level ( $r=-0.569, p<0.001)$, d) C-reactive protein (CRP) level and erythrocyte sedimentation rate (ESR), and e) radiologically affected lobes. The difference in f) disease severity (classified as mild, common, severe or critical, according to the guidelines from the National Health Commission of China [8], but with no mild patients in our cohort), g) treatment, and h) disease course and rate of respiratory failure in the two age groups were also investigated. Cure rates of COVID-19 are shown for i) all 221 patients, j) males, k) females and (I) those with respiratory failure. ${ }^{*}$ : $p<0.05 ;{ }^{* *}: p<0.01 ;{ }^{* * *}: p<0.001$.

This is the first study to systematically evaluate the impact of age on the clinical characteristics and important outcomes for COVID-19 patients, thus helping clinicians to establish risk stratification of COVID-19 patients as early as possible. Sporadic studies have mentioned that elderly people may tend to die after infection $[5,7,9]$, calling on the public to pay more attention to protecting the elderly from the virus. In this study, we demonstrated that the clinical characteristics and outcomes of 221 COVID-19 patients were closely related to the different age groups. This study provides clear evidence of a relationship between disease severity and age, which other studies have not. Comprehensive analysis of 
these indicators will provide physicians worldwide with important information for disease perception, condition assessment and effective treatments for COVID-19.

The proportion of patients using antibiotics was higher in patients aged $\geqslant 60$ years than in patients aged $<60$ years, possibly due to the higher proportion of patients with bacterial co-infection in this group. Besides, older patients showed more serious illness, leading to higher frequency of adjuvant therapies including corticosteroids and assisted ventilation in this group of patients. Consistent with recent reports $[10,11]$, older patients presented significantly lower levels of lymphocytes than younger patients. Lymphocytes are generally elevated in response to common viral infections, but are abnormally decreased in severe acute respiratory syndrome (SARS) and COVID-19 [12-14]. Although the underlying mechanism is still unclear, the low level of lymphocytes could be a key indicator of disease severity in COVID-19. Furthermore, other serological indexes, such as albumin level, blood urea nitrogen, lactate dehydrogenase and inflammatory indicators, also showed a progressive trend with age. The phenomenon is obvious; however, we cannot identify whether these changed indicators result in or result from the differences in disease severity with age.

Although most patients had favourable prognosis in this study, some patients required longer periods of treatment, which might have turned out worse due to the high risk of hospital-acquired or iatrogenic infections. Age was one of the risk factors for disease severity and mortality in viral infection studies [5, 9], although the data about age in those studies are approximate. Our study found that COVID-19 patients aged $\geqslant 60$ years had a higher rate of respiratory failure and needed more prolonged treatment than those aged $<60$ years, demonstrating that elderly COVID-19 patients had much more severe disease and showed poorer response to treatments than the younger age group. The cure rate of patients aged $\geqslant 60$ years $(89.4 \%)$ was relatively lower than that of patients aged $<60$ years $(95.6 \%)$, especially in males and those with respiratory failure. A previous study indicated that there might be a sex predisposition to COVID-19, with men more prone to be affected, but gave no evidence of an association between the severity of COVID-19 and the male sex [15]. More attention needs to be paid to these older patients with respiratory failure, and aggressive early intervention should be made to improve their prognosis. With more cases being examined from different ethnic and genetic backgrounds, the findings related to age in this study may be confirmed by physicians worldwide.

In conclusion, the clinical features and prognosis of the disease vary among patients of different ages and a thorough assessment of age may help clinicians worldwide to establish risk stratification for all COVID-19 patients. Patients aged $\geqslant 60$ years showed heavier clinical manifestations, greater severity and longer disease courses compared with those aged $<60$ years. Closer monitoring and more medical interventions may be needed for the elderly.

Yang Liu'1,13, Bei Mao ${ }^{1,13}$, Shuo Liang ${ }^{1,13}$, Jia-Wei Yang ${ }^{1,13}$, Hai-Wen $\mathrm{Lu}^{1,13}$, Yan-Hua Chai ${ }^{1,13}$, Lan Wang ${ }^{2}$, Li Zhang ${ }^{1}$, Qiu-Hong $\mathrm{Li}^{1}$, Lan Zhao ${ }^{1}$, Yan $\mathrm{He}^{1}$, Xiao-Long Gu${ }^{3}$, Xiao-Bin $\mathrm{i}^{4}$, Li Li ${ }^{5}$, Zhi-Jun Jie ${ }^{6}$, Qiang Li ${ }^{7}$, XiangYang $\mathrm{Li}^{8}$, Hong-Zhou Lu', Wen-Hong Zhang ${ }^{10}$, Yuan-Lin Song ${ }^{11}$, Jie-Ming $\mathrm{Qu}^{12,14}$ and Jin-Fu Xu ${ }^{1,14}$ on behalf of the Shanghai Clinical Treatment Experts Group for COVID-19

${ }^{1}$ Dept of Respiratory and Critical Care Medicine, Shanghai Pulmonary Hospital, Tongji University School of Medicine, Shanghai, China. ${ }^{2}$ Dept of Pulmonary Circulation Medicine, Shanghai Pulmonary Hospital, Tongji University School of Medicine, Shanghai, China. ${ }^{3}$ Dept of Respiratory Medicine, Shanghai Pudong New Area People's Hospital, Shanghai, China. ${ }^{4}$ Dept of Respiratory Medicine, Zhoupu Hospital of Pudong New District, Shanghai, China. ${ }^{5}$ Dept of Respiratory Medicine, Baoshan District Hospital of Integrated Traditional Chinese and Western Medicine, Shanghai, China. ${ }^{6}$ Dept of Respiratory and Critical Care Medicine, Shanghai Fifth People's Hospital, Fudan University, Shanghai, China. ${ }^{7}$ Dept of Respiratory and Critical Care Medicine, Shanghai East Hospital, Tongji University, Shanghai, China. ${ }^{8}$ Dept of Respiratory and Critical Care Medicine, Huadong Hospital, Fudan University, Shanghai, China. ${ }^{9}$ Shanghai Public Health Clinical Center, Shanghai, China. ${ }^{10}$ Dept of Infectious Disease, Huashan Hospital Fudan University, Shanghai, China. ${ }^{11}$ Dept of Respiratory and Critical Care Medicine, Zhongshan Hospital Fudan University, Shanghai, China. ${ }^{12}$ Dept of Respiratory and Critical Care Medicine, Ruijin Hospital, Shanghai Jiaotong University School of Medicine, Shanghai, China. ${ }^{13}$ These authors contributed equally. ${ }^{14}$ These authors contributed equally.

Correspondence: Jin-Fu Xu, Dept of Respiratory and Critical Care Medicine, Shanghai Pulmonary Hospital, Tongji University School of Medicine, No. 507 Zhengmin Road, Shanghai, China. E-mail: jfxucn@163.com

Received: 20 March 2020 | Accepted: 11 April 2020

Acknowledgements: The authors would like to express sincere thanks to all the staff of all the hospitals, and also to the patients for their contributions to the study.

Author contributions: Jin-Fu Xu and Jie-Ming Qu conceived and designed the study. Yang Liu, Bei Mao, Shuo Liang, Hai-Wen $\mathrm{Lu}$, Yan-Hua Chai and Jin-Fu Xu conducted the primary analysis and prepared the first draft of the manuscript. Lan Wang, Li Zhang, Qiu-Hong Li, Lan Zhao, Yan He, Xiao-Long Gu, Xiao-Bin Ji, Li Li, Zhi-Jun Jie, Qiang Li, Xiang-Yang Li, Hong-Zhou Lu, Wen-Hong Zhang and Yuan-Lin Song reviewed the draft for intellectual content. The corresponding authors Jin-Fu Xu and Jie-Ming $\mathrm{Qu}$ had full access to all the data in the study and take responsibility for the integrity of the data and the accuracy of the data analysis. 
Conflict of interest: None declared.

Support statement: This work was supported by the National Natural Science Fund for Distinguished Young Scholars to Jin-Fu Xu (81925001), Shanghai Leading Talent Program (number 2016036 to Jin-Fu Xu) and the Project of the Shanghai Hospital Development Center (16CR3036A to Jin-Fu Xu). The funders had no role in the design and conduct of the study; collection, management, analysis and interpretation of the data; review or approval of the manuscript; and decision to submit the manuscript for publication. No authors have been paid to write this article by any pharmaceutical companies or agencies. Funding information for this article has been deposited with the Crossref Funder Registry.

\section{References}

1 Burki T. Outbreak of coronavirus disease 2019. Lancet Infect Dis 2020; 20: 292-293.

2 Guan WJ, Chen RC, Zhong NS. Strategies for the prevention and management of coronavirus disease 2019. Eur Respir J 2020; 55: 2000597.

3 Miller EJ, Linge HM. Age-related changes in immunological and physiological responses following pulmonary challenge. Int J Mol Sci 2017; 18: E1294.

4 Yang X, Yu Y, Xu J, et al. Clinical course and outcomes of critically ill patients with SARS-CoV-2 pneumonia in Wuhan, China: a single-centered, retrospective, observational study. Lancet Respir Med 2020; 8: 475-481.

5 Zhou F, Yu T, Du R, et al. Clinical course and risk factors for mortality of adult inpatients with COVID-19 in Wuhan, China: a retrospective cohort study. Lancet 2020; 395: 1054-1062.

$6 \mathrm{Wu}$ C, Chen X, Cai Y, et al. Risk factors associated with acute respiratory distress syndrome and death in patients with coronavirus disease 2019 pneumonia in Wuhan, China. JAMA Intern Med 2020; in press [https://doi.org/10. 1001/jamainternmed.2020.0994].

7 Du RH, Liang LR, Yang CQ, et al. Predictors of mortality for patients with COVID-19 pneumonia caused by SARS-CoV-2: a prospective cohort study. Eur Respir J 2020; 55: 2000524.

8 Lin L, Li TS. [Interpretation of "Guidelines for the diagnosis and treatment of novel coronavirus (2019-nCoV) infection by the National Health Commission (Trial Version 5)"]. Zhonghua Yi Xue Za Zhi 2020; 100: 805-807.

9 Wang W, Tang J, Wei F. Updated understanding of the outbreak of 2019 novel coronavirus (2019-nCoV) in Wuhan, China. J Med Virol 2020; 92: 441-447.

10 Wang $\mathrm{D}, \mathrm{Hu}$ B, Hu C, et al. Clinical characteristics of 138 hospitalized patients with 2019 novel coronavirus-infected pneumonia in Wuhan, China. JAMA 2020; 323: 1061-1069.

11 Guan WJ, Ni ZY, Hu Y, et al. Clinical characteristics of coronavirus disease 2019 in China. N Engl J Med 2020; 382: $1708-1720$.

12 He Z, Zhao C, Dong Q, et al. Effects of severe acute respiratory syndrome (SARS) coronavirus infection on peripheral blood lymphocytes and their subsets. Int J Infect Dis 2005; 9: 323-330.

13 Zhang JJ, Dong X, Cao YY, et al. Clinical characteristics of 140 patients infected with SARS-CoV-2 in Wuhan, China. Allergy 2020; in press [https://doi.org/10.1111/all.14238].

$14 \mathrm{Xu} \mathrm{YH}$, Dong JH, An WM, et al. Clinical and computed tomographic imaging features of novel coronavirus pneumonia caused by SARS-CoV-2. J Infect 2020; 80: 394-400.

15 Cai H. Sex difference and smoking predisposition in patients with COVID-19. Lancet Respir Med 2020; 8: e20. 